\title{
Image Superresolution Reconstruction via Granular Computing Clustering
}

\author{
Hongbing Liu, Fan Zhang, Chang-an Wu, and Jun Huang \\ School of Computer and Information Technology, Xinyang Normal University, Xinyang 464000, China \\ Correspondence should be addressed to Hongbing Liu; liuhbing@126.com
}

Received 18 August 2014; Revised 21 October 2014; Accepted 23 November 2014; Published 28 December 2014

Academic Editor: Yuanqing Li

Copyright ( 2014 Hongbing Liu et al. This is an open access article distributed under the Creative Commons Attribution License, which permits unrestricted use, distribution, and reproduction in any medium, provided the original work is properly cited.

\begin{abstract}
The problem of generating a superresolution (SR) image from a single low-resolution (LR) input image is addressed via granular computing clustering in the paper. Firstly, and the training images are regarded as SR image and partitioned into some SR patches, which are resized into LS patches, the training set is composed of the SR patches and the corresponding LR patches. Secondly, the granular computing $(\mathrm{GrC})$ clustering is proposed by the hypersphere representation of granule and the fuzzy inclusion measure compounded by the operation between two granules. Thirdly, the granule set (GS) including hypersphere granules with different granularities is induced by $\mathrm{GrC}$ and used to form the relation between the LR image and the SR image by lasso. Experimental results showed that GrC achieved the least root mean square errors between the reconstructed SR image and the original image compared with bicubic interpolation, sparse representation, and NNLasso.
\end{abstract}

\section{Introduction}

In the field of image processing, SR images are usually desired for later image processing and analysis. Improvement of picture information for human interpretation and helping representation for automatic machine perception are two principal application areas [1]. The resolution of a digital image can be classified in many different ways: pixel resolution, spatial resolution, spectral resolution, temporal resolution, and radiometric resolution [2-5].

An image of $N$ pixels height by $M$ pixels width can have any resolution less than $N$ lines per picture height. But when the pixel counts are referred to as resolution, the convention is to describe the pixel resolution with the set of two positive integer numbers, where the first number is the number of pixel columns (width) and the second is the number of pixel rows (height), for example, as 7680 by 6876 . Another popular convention is to cite resolution as the total number of pixels in the image, typically given as number of megapixels, which can be calculated by multiplying pixel columns by pixel rows and dividing by one million. Other conventions include describing pixels per length unit or pixels per area unit, such as pixels per inch or per square inch. None of these pixel resolutions are true resolutions, but they are widely referred to as such; they serve as upper bounds on image resolution [2].

SR is a technique that constructs high-resolution (HR) images from several observed LR images, thereby increasing the high frequency components and removing the degradations caused by the imaging process of the LR camera. The basic idea behind SR is to combine the nonredundant information contained in multiple LR frames to generate a HR image. A closely related technique with SR is the single image interpolation approach, which can be also used to increase the image size $[1,5]$. However, since there is no additional information provided, the quality of the single image interpolation is very much limited due to the ill-posed nature of the problem, and the lost frequency components cannot be recovered. In the SR setting, however, multiple LR observations are available for reconstruction, making the problem better constrained. The nonredundant information contained in these LR images is typically introduced by subpixel shifts between them. These subpixel shifts may occur due to uncontrolled motions between the imaging system and scene, for example, movements of objects, or due to controlled motions, for example, the satellite imaging system orbiting the earth with predefined speed and path $[6,7]$. 
$\mathrm{GrC}$ is a transformation method between the universe and the parts and is widely used in pattern recognition, information system, and so forth. Zadeh identified three fundamental concepts of the human cognition process, namely, granulation, organization, and causation $[8,9]$. Granulation is a process that decomposes a universe into parts. Conversely, organization is a process that integrates parts into a universe by introducing operation between two granules. Causation involves the association of causes and effects. The construction of granule set, the operations between two granules, and the inclusion measure between two granules are main researches in GrC. In general, the fuzzy inclusion measure is induced by granule and union granule, such that the positive valuation functions of granules are used to define the fuzzy inclusion measure [10-15].

The present work uses GrC clustering to construct the SR image of the original image. The granules are represented as the hypersphere, and the join operation and meet operation are designed to obtain GS including the granules with different granularities. The fuzzy inclusion measures are compounded by the positive valuation functions.

The rest of this paper is presented as follows. The theoretical background of GrC clustering is described in Section 2. Section 3 designs the $\mathrm{GrC}$ clustering algorithms. The image SR reconstruction experiments are used to demonstrate $\mathrm{GrC}$ clustering in Section 4. Section 5 summarizes the contribution of our work and presents future work plans.

\section{Theoretical Background}

GrC clustering is discussed on the basis of the contribution of Kaburlasos and his colleagues in the view of set theory [1015].

2.1. Representation of Granules. A granule is represented as a subset of $S$ which is composed by the data with the similar features, and the size of granule is measured by the granularity defined by the maximal distance between data belonging to the same granule. In order to facilitate the study of granular computing, such as the operations between two granules, the granules are represented as the standard form, for example, the granule with the shape of circle in 2-dimensional space and the shape of hypersphere in $\mathrm{N}$-dimensional space.

A granule is represented as the hypersphere $\mathbf{G}=(\mathbf{C}, R)$, where $\mathbf{C}$ is the center of granule and $R$ is radius of granule, and refers to the granularity of granule $\mathbf{G}$ which is measured by the maximal distance between center and the data included in granule. Particularly, a point $\mathbf{x}$ is represented by an atomic granule with the center $\mathbf{x}$ and granularity 0 in $N$-dimensional space. The distance between center $\mathbf{C}=\left(c_{1}, c_{2}, \ldots, c_{N}\right)$ and datum $\mathbf{x}=\left(x_{1}, x_{2}, \ldots, x_{N}\right)$ can be defined as follows:

$$
\begin{aligned}
& d(\mathbf{x}, \mathbf{C}) \\
& \quad=\left(\left(x_{1}-c_{1}\right)^{2}+\left(x_{2}-c_{2}\right)^{2}+\cdots+\left(x_{N}-c_{N}\right)^{2}\right)^{1 / 2} .
\end{aligned}
$$

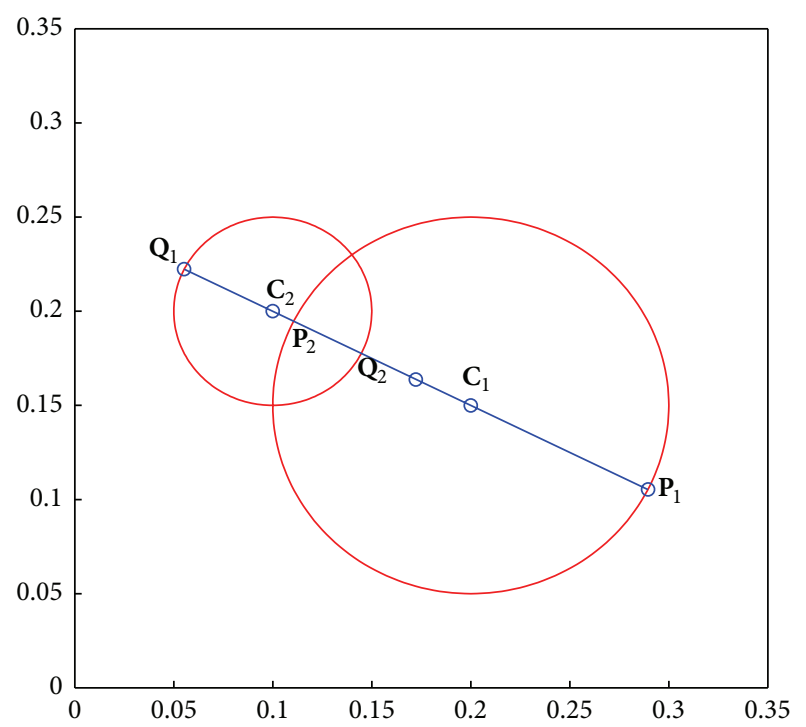

FIGURE 1: The cross-points between hypersphere granules and the line through $\mathbf{C}_{12} . \mathbf{P}_{1}$ and $\mathbf{P}_{2}$ are the cross-points between hypersphere $\mathbf{G}_{1}=\left(\mathbf{C}_{1}, R_{1}\right)$ and the line through $\mathbf{C}_{1}$ and $\mathbf{C}_{2}$, and $\mathbf{Q}_{1}$ and $\mathbf{Q}_{2}$ are the cross-points between hypersphere $\mathbf{G}_{2}=\left(\mathbf{C}_{2}, R_{2}\right)$ and the line through $\mathbf{C}_{1}$ and $\mathbf{C}_{2}$.

2.2. Operations between Two Granules. The operations between two granules reflect the transformation between the macro world and the microscopic world of human cognition. When a person wants to observe the object more carefully, the object is partitioned into some suitable subobjects; namely, the universe is partitioned into some parts in order to study the object in detail in the view of the microscopic world. Conversely, if some objects have the same attributes, we regard them as a universe in the view of the macro world. The operations between two granules are designed to realize the transformation between the macro world and the microscopic world. Set-based models of granular structures are special cases of lattice-based models, where the lattice join operation $V$ coincides with set union operation $U$ and lattice meet operation $\wedge$ coincides with set intersection operation $\cap$.

Join operation $\vee$ and meet operation $\wedge$ are used to realize the transformation between the macro world and the microscopic world. Operation $\vee$ unites the granules with small granularities to the granules with the large granularities. Inversely, operation $\wedge$ divides the granules with large granularities into the granules with small granularities. Join operation is associated with the dilation operation of mathematical morphology (MM), and meet operation is associated with the erosion operation of MM [15]. In image analysis field, dilation operation replaces all the pixel values in the neighborhood with the maximal pixel value, and erosion operation replaces all the pixel values in the neighborhood with the minimal pixel value [16]. Join operation $\vee$ and meet operation $\wedge$ are designed as follows. 


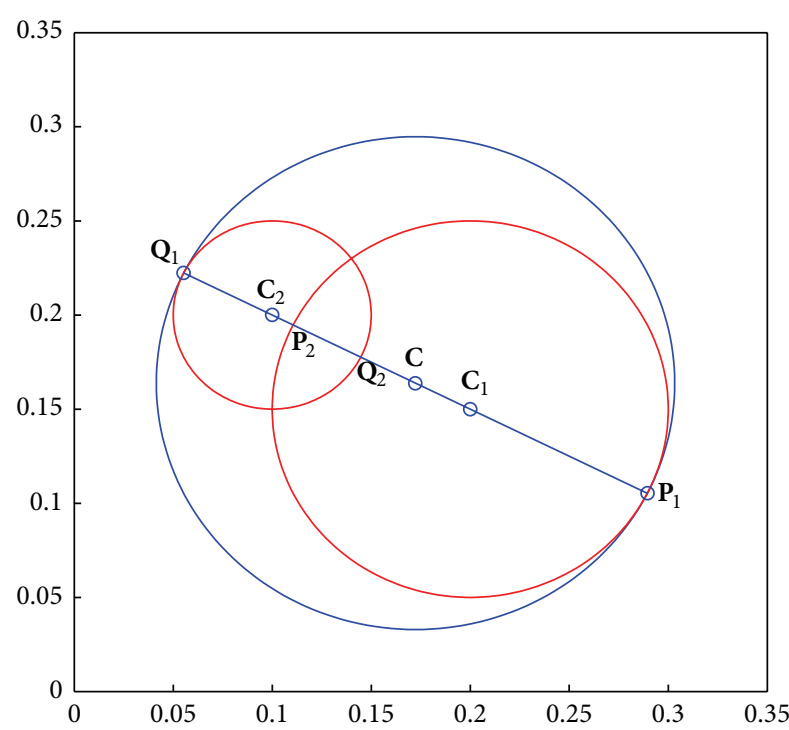

Figure 2: The join hypersphere granule between two hypersphere granules. The hypersphere with blue curve is the join hypersphere between two hyperspheres with red curves.

All points are regarded as atomic granules which are indivisible, and the join process is the key to obtain the larger granules compared with atomic granules. Likewise, the whole space is a granule with the maximal granularity, and the meet process produces the smaller granules compared with original granules.

For two hypersphere granules $\mathbf{G}_{1}=\left(\mathbf{C}_{1}, R_{1}\right)$ and $\mathbf{G}_{2}=\left(\mathbf{C}_{2}, R_{2}\right)$ in $N$-dimensional space, the join hypersphere granule is

$$
\mathbf{G}=\mathbf{G}_{1} \vee \mathbf{G}_{2}=(\mathbf{C}, R) .
$$

The center $\mathbf{C}$ of $\mathbf{G}$ and the granularity of $\mathbf{G}$ are computed as follows.

Firstly, the vector from $\mathrm{C}_{1}$ to $\mathrm{C}_{2}$ and vector from $\mathrm{C}_{2}$ to $\mathbf{C}_{1}$ are computed. If $\mathbf{C}_{1}=\mathbf{C}_{2}$, then $\mathbf{C}_{12}=0$ and $\mathbf{C}_{21}=0$. If $\mathbf{C}_{1} \neq \mathbf{C}_{2}$, then $\mathbf{C}_{12}=\left(\mathbf{C}_{2}-\mathbf{C}_{1}\right) / d\left(\mathbf{C}_{1}, \mathbf{C}_{2}\right)$ and $\mathbf{C}_{21}=\left(\mathbf{C}_{1}-\right.$ $\left.\mathbf{C}_{2}\right) / d\left(\mathbf{C}_{2}, \mathbf{C}_{1}\right)$

Secondly, the cross-points between the hypersphere $\mathbf{G}_{1}$ and the line through $\mathbf{C}_{12}$ are $\mathbf{P}_{1}=\mathbf{C}_{1}-\mathbf{C}_{12} R_{1}$ and $\mathbf{P}_{2}=\mathbf{C}_{1}+$ $\mathrm{C}_{12} R_{1}$. The cross-points between the hypersphere $\mathrm{G}_{2}$ and the line through $\mathbf{C}_{12}$ are $\mathbf{Q}_{1}=\mathbf{C}_{2}-R_{2} \mathbf{C}_{21}$ and $\mathbf{Q}_{2}=\mathbf{C}_{2}+R_{2} \mathbf{C}_{21}$. The cross-points are shown in Figure 1.

Thirdly, the join hypersphere granule $\mathbf{G}$ is computed by the following formulas:

$$
\begin{aligned}
\mathbf{G} & =\mathbf{G}_{1} \vee \mathbf{G}_{2} \\
& =\left\{\begin{array}{c}
\mathbf{C}=\mathbf{C}_{1}, \quad R=R_{1} \\
\text { if } R_{1} \geq R_{2}, \quad d\left(\mathbf{C}_{1}, \mathbf{C}_{2}\right) \leq R_{1}-R_{2} \\
\mathbf{C}=\mathbf{C}_{2}, \quad R=R_{2} \\
\text { if } R_{1}<R_{2}, \quad d\left(\mathbf{C}_{1}, \mathbf{C}_{2}\right) \leq R_{2}-R_{1} \\
\mathbf{C}=0.5\left(\mathbf{P}_{1}+\mathbf{Q}_{1}\right), \quad R=0.5 d\left(\mathbf{P}_{1}, \mathbf{Q}_{1}\right) \\
\text { if } d\left(\mathbf{C}_{1}, \mathbf{C}_{2}\right)>\left|R_{1}-R_{2}\right| .
\end{array}\right.
\end{aligned}
$$

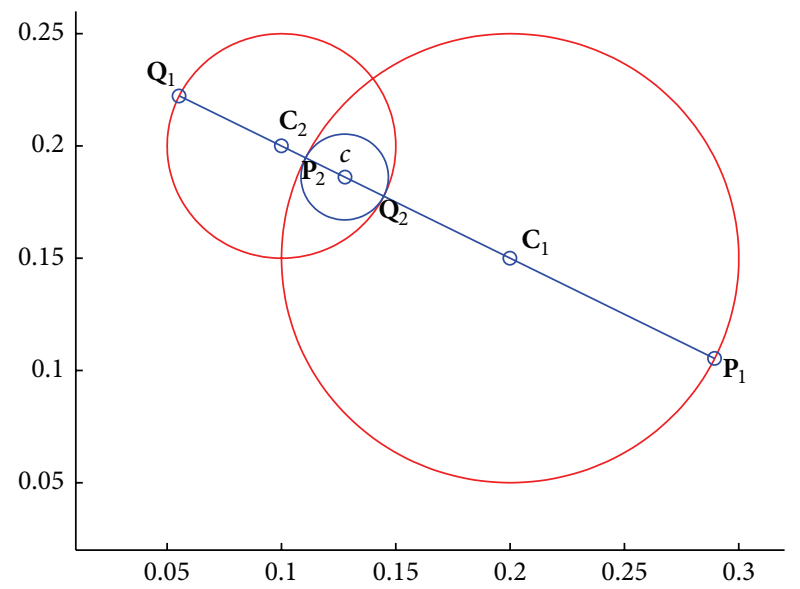

FIGURE 3: The meet hypersphere granule between two hypersphere granules. The hypersphere with blue curve is the meet hypersphere between two hyperspheres with red curves.

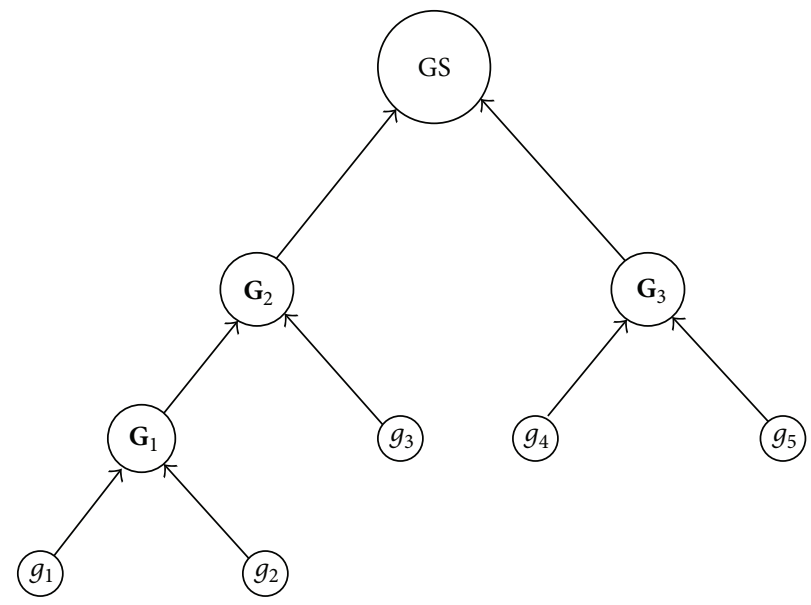

FIGURE 4: The clustering process of TS including 5 samples. Leafs denote the atomic hypersphere granules, branch points denote the join hypersphere granule, and the tree root denotes GS.

Similarly, the meet hypersphere granule is computed:

$$
\begin{aligned}
g & =\mathbf{G}_{1} \wedge \mathbf{G}_{2} \\
& =\left\{\begin{array}{l}
\varnothing \\
\quad \text { if } d\left(\mathbf{C}_{1}, \mathbf{C}_{2}\right)>R_{1}+R_{2} \\
c=\mathbf{C}_{2}, \quad r=R_{2} \\
\text { if } d\left(\mathbf{C}_{1}, \mathbf{C}_{2}\right)<R_{1}-R_{2}, \quad R_{1} \geq R_{2} \\
c=\mathbf{C}_{1}, \quad r=R_{1} \\
\text { if } d\left(\mathbf{C}_{1}, \mathbf{C}_{2}\right)<R_{2}-R_{1}, \quad R_{2} \geq R_{1} \\
c=0.5\left(\mathbf{P}_{2}+\mathbf{Q}_{2}\right), \quad r=0.5 d\left(\mathbf{P}_{2}, \mathbf{Q}_{2}\right) \\
\text { if } d\left(\mathbf{C}_{1}, \mathbf{C}_{2}\right)>\left|R_{1}-R_{2}\right| .
\end{array}\right.
\end{aligned}
$$

2.3. Fuzzy Inclusion Measure. As mentioned above, for all $\mathbf{G}_{1}, \mathbf{G}_{2} \in \mathrm{GS}, \mathbf{G}_{1} \subseteq \mathbf{G}_{1} \vee \mathbf{G}_{2}$, and $\mathbf{G}_{2} \subseteq \mathbf{G}_{1} \vee \mathbf{G}_{2}, \mathbf{G}_{1} \wedge \mathbf{G}_{2} \subseteq \mathbf{G}_{1}$ and $\mathbf{G}_{1} \wedge \mathbf{G}_{2} \subseteq \mathbf{G}_{2}$. Namely, the operations between granule 

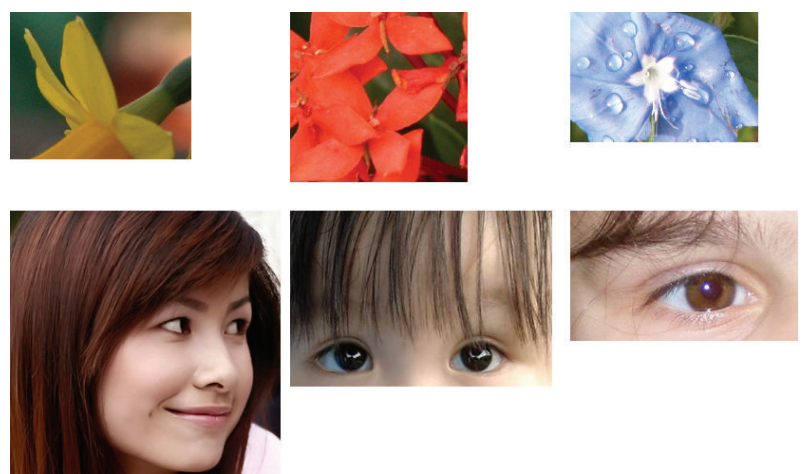

FIGURE 5: Training images with different sizes.

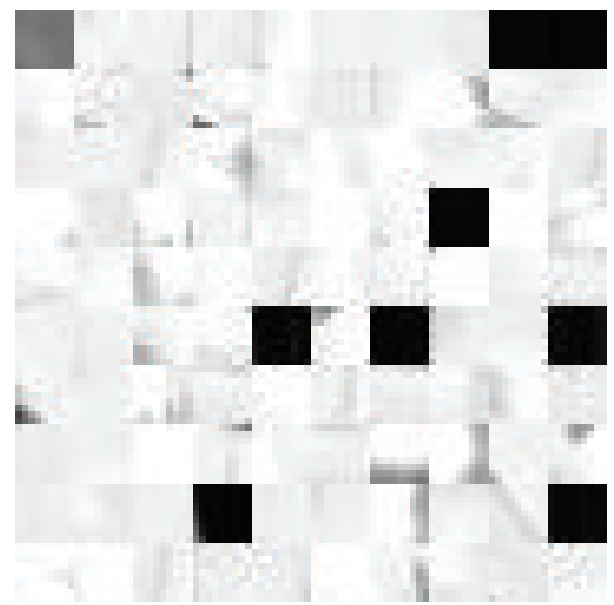

Figure 6: The patches sampled by GrC with $\rho=100$ from the training image shown in Figure 5.

$\mathbf{G}_{1}$ and granule $\mathbf{G}_{2}$ are corresponding to the inclusion relation between granules $\mathbf{G}_{1}$ and $\mathbf{G}_{2}$ :

$$
\begin{aligned}
\mathbf{G}_{1} & \subseteq \mathbf{G}_{2} \Longleftrightarrow \mathbf{G}_{1} \vee \mathbf{G}_{2}=\mathbf{G}_{2}, \\
\mathbf{G}_{1} \wedge \mathbf{G}_{2} & =\mathbf{G}_{1} .
\end{aligned}
$$

The inclusion relation between two hypersphere granules is induced by the operations between two hypersphere granules.

The join hypersphere granule and the meet hypersphere granule are used to measure the fuzzy inclusion relation. The granularity $R$ is used to define the fuzzy inclusion measure:

$$
\begin{aligned}
K\left(\mathbf{G}_{1}, \mathbf{G}_{2}\right) & =\frac{v\left(\mathbf{G}_{2}\right)}{v\left(\mathbf{G}_{1} \vee \mathbf{G}_{2}\right)}, \\
S\left(\mathbf{G}_{1}, \mathbf{G}_{2}\right) & =\frac{v\left(\mathbf{G}_{1} \wedge \mathbf{G}_{2}\right)}{v\left(\mathbf{G}_{2}\right)},
\end{aligned}
$$

where $v(\mathbf{G})$ is the positive valuation function defined by Kaburlasos et al., which can be the linear function or nonlinear function [10-13]. A valuation function $v: L \rightarrow R$ is a mapping between a lattice $L$ and a real number. The valuation function satisfies $v(a)+v(b)=v(a \wedge b)+v(a \vee b)$, $a, b \in L$. A valuation function is called positive if and only if $a<b \Rightarrow v(a)<v(b)[14]$.

The hypersphere granule set is a mathematical lattice if the inclusion measure is defined as (6a) and (6b). More specifically, (6a) and (6b) can be used for hyperspheres based on the lattice of intervals on the line defined by the centers $\mathbf{C}_{1}$ and $\mathbf{C}_{2}$ of the hyperspheres $\left(\mathbf{C}_{1}, R_{1}\right)$ and $\left(\mathbf{C}_{2}, R_{2}\right)$, respectively, as explained in Example 2.8 in [11].

According to [15], the strictly increasing function is a positive valuation. For $\mathbf{G}=(\mathbf{C}, R)$,

$$
v(\mathbf{G})=R+\beta
$$

is a positive valuation function defined on GS, where $\beta$ is a constant.

2.4. Fuzzy Algebraic Structures. For a training set $S=$ $\left\{\mathbf{x}_{i} \mid i=1,2, \ldots, n\right\}$, every datum $x_{i}$ is represented as an atomic hypersphere granule which is indivisible, and the granule set is obtained. For the positive valuation function (7), the fuzzy inclusion relation between two hypersphere granules is computed by formulas (6a) and (6b). So the fuzzy algebraic structures $\left\langle\mathrm{GS}, K\left(\mathbf{G}_{1}, \mathbf{G}_{2}\right)\right\rangle$ and $\left\langle\mathrm{GS}, S\left(\mathbf{G}_{1}, \mathbf{G}_{2}\right)\right\rangle$ are formed by $G S$ and $K\left(\mathbf{G}_{1}, \mathbf{G}_{2}\right)$, where $K\left(\mathbf{G}_{1}, \mathbf{G}_{2}\right)$ implies the operation between two hypersphere granules and $S\left(\mathbf{G}_{1}, \mathbf{G}_{2}\right)$ implies the meet operation between two hypersphere granules. $\left\langle\mathrm{GS}, K\left(\mathbf{G}_{1}, \mathbf{G}_{2}\right)\right\rangle$ and $\left\langle\mathrm{GS}, S\left(\mathbf{G}_{1}, \mathbf{G}_{2}\right)\right\rangle$ are proved as fuzzy lattice, and $K\left(\mathbf{G}_{1}, \mathbf{G}_{2}\right)$ and $S\left(\mathbf{G}_{1}, \mathbf{G}_{2}\right)$ are fuzzy inclusion measures, which satisfied the following four conditions [12, $14,17]$.

(1) If $\mathbf{G} \neq \varnothing$, then $K(\mathbf{G}, \varnothing)=0, S(\mathbf{G}, \varnothing)=0$.

(2) For $\mathbf{G} \in \mathrm{GS}, K(\mathbf{G}, \mathbf{G})=1, S(\mathbf{G}, \mathbf{G})=1$.

(3) If $\mathbf{G}_{1} \leq \mathbf{G}_{2}$, then $K\left(\mathbf{G}, \mathbf{G}_{1}\right) \leq K\left(\mathbf{G}, \mathbf{G}_{2}\right) S\left(\mathbf{G}, \mathbf{G}_{1}\right) \leq$ $S\left(\mathbf{G}, \mathbf{G}_{2}\right)$.

(4) If $\mathbf{G}_{1} \wedge \mathbf{G}_{2}<\mathbf{G}_{1}$, then $K\left(\mathbf{G}_{1}, \mathbf{G}_{2}\right)<1$.

\section{GrC Clustering}

For the data set $S=\left\{\mathbf{x}_{i} \mid i=1,2, \ldots, n\right\}$ in $N$-dimensional space, we form the following three algorithms based on the aforementioned theoretical background.

Algorithm 1 is the join process between two hypersphere granules and produces the hypersphere granule with the larger granularity compared with the original hypersphere granules. For example, the join hypersphere of hypersphere granules $\mathbf{G}_{1}=[0.2,0.15,0.1]$ and $\mathbf{G}_{2}=[0.1,0.2,0.05]$ in 2dimensional space is $\mathbf{G}=[0.1724,0.1638,0.1309]$ as shown in Figure 2.

Algorithm 2 is the meet process between two hypersphere granules and produces the hypersphere granule with the smaller granularity compared with the original hypersphere granules. The meet process of hypersphere granule $\mathbf{G}_{1}=[0.2$, $0.15,0.1]$ and hypersphere granule $\mathbf{G}_{2}=[0.1,0.2,0.0 .05]$ is $g$ $=[0.1276,0.1862,0.0191]$ as shown in Figure 3 .

For data set $S$, the GrC clustering algorithms are proposed based on the join process by the following steps. Firstly, the samples are used to form the atomic granule. Secondly, 


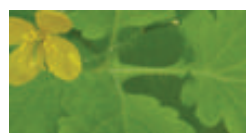

(a)

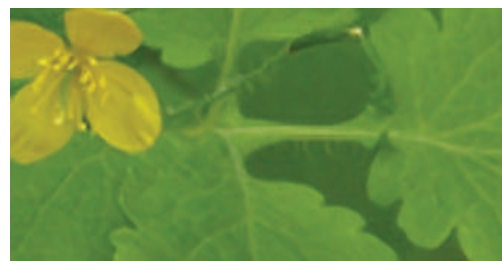

(d)

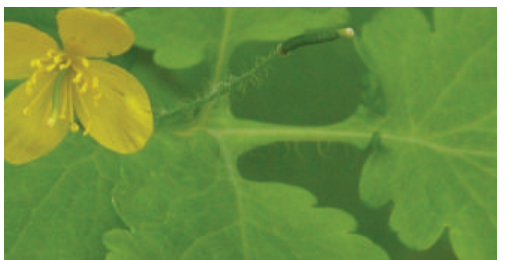

(b)

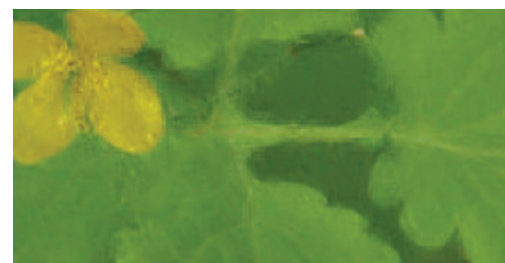

(e)

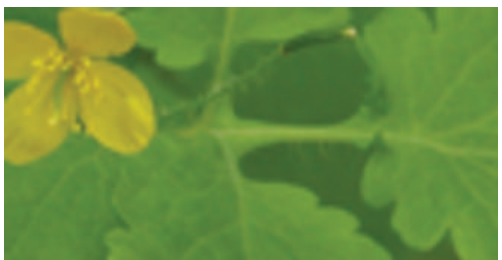

(c)

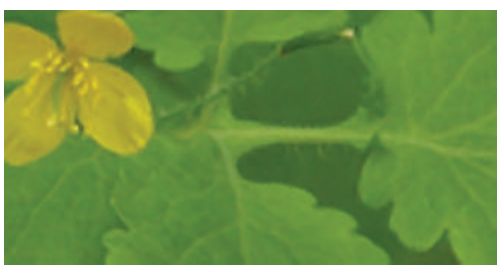

(f)

FIGURE 7: The flower image magnified by a factor of 3. Left to right: (a) low-resolution image, (b) the original image, (c) superresolution image by bicubic interpolation, (d) superresolution image by sparse representation, (e) superresolution image by NNLasso, and (f) superresolution image by GrC.

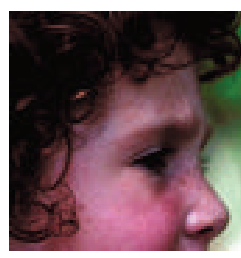

(a)

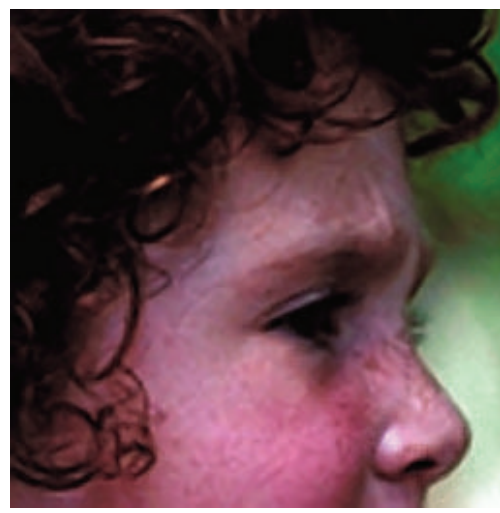

(d)

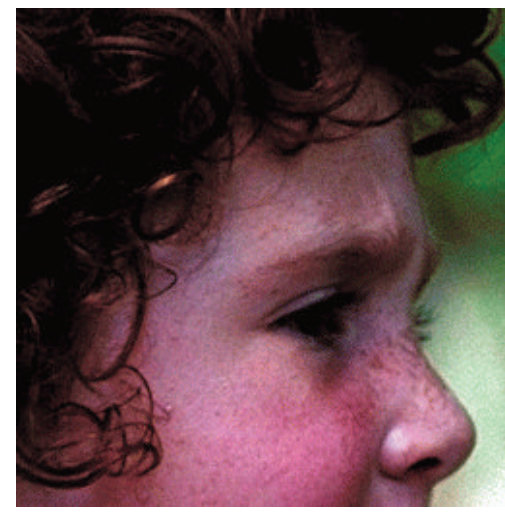

(b)

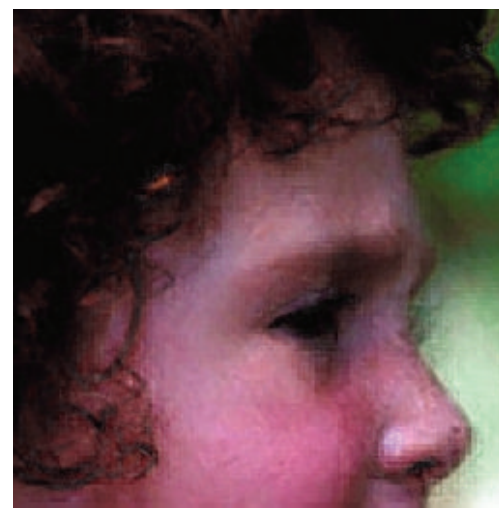

(e)

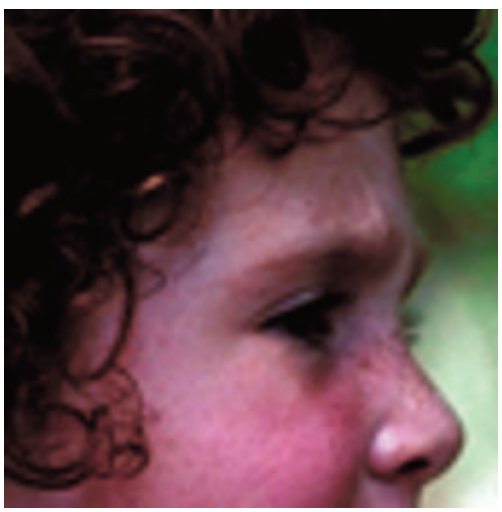

(c)

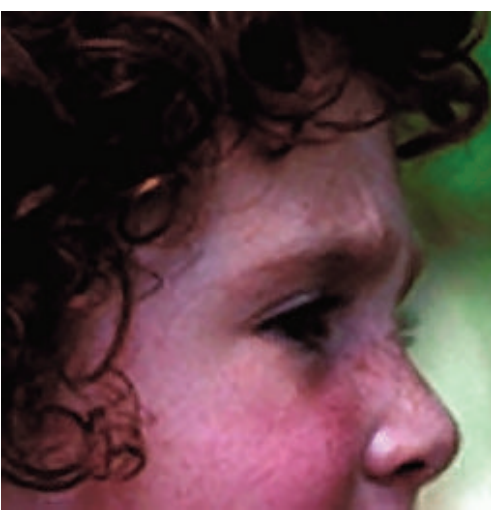

(f)

Figure 8: The girl image magnified by a factor of 3. (a) Low-resolution image, (b) the original image, (c) superresolution image by bicubic interpolation, (d) superresolution image by sparse representation, (e) superresolution image by NNLasso, and (f) superresolution image by GrC. 


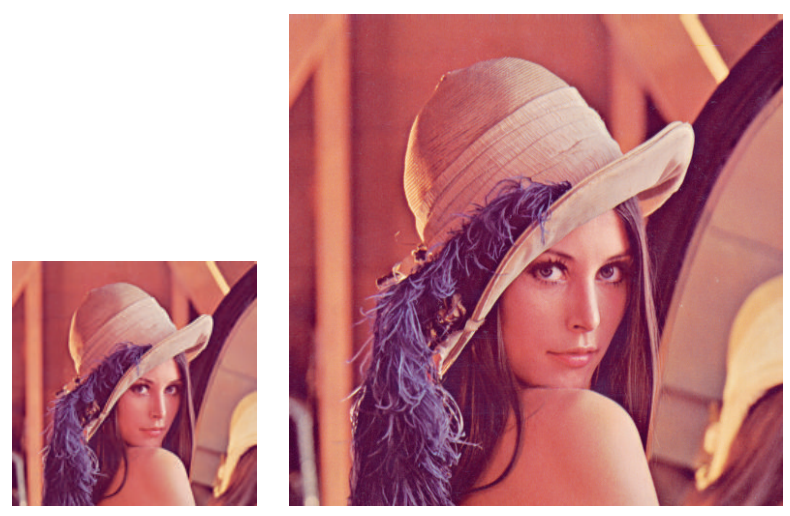

(a)

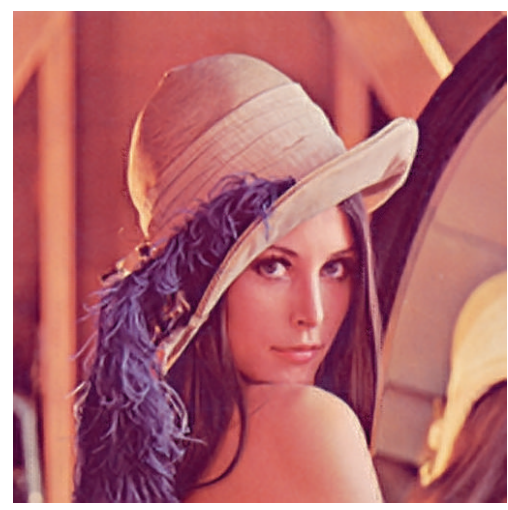

(d) (b)

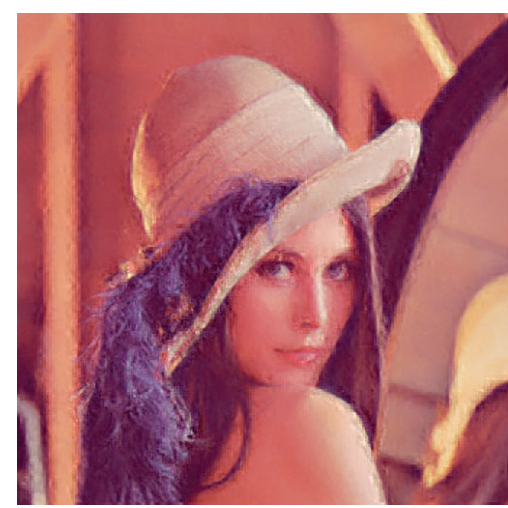

(e)

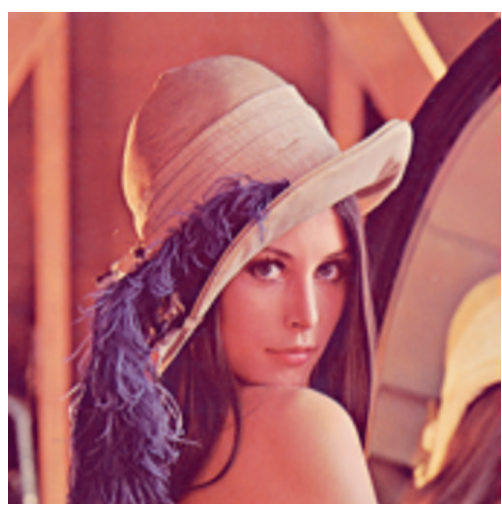

(c)

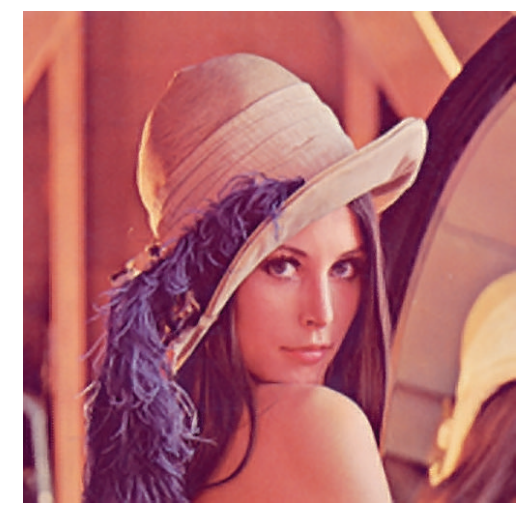

(f)

FiguRE 9: The Lenna image magnified by a factor of 3. (a) Low-resolution image, (b) the original image, (c) superresolution image by bicubic interpolation, (d) superresolution image by sparse representation, (e) superresolution image by NNLasso, and (f) superresolution image by GrC.

the threshold of granularity is introduced to conditionally unite the atomic granules by the aforementioned join operation, and the granule set is composed of all the join granules. Thirdly, if all atomic granules are included in the granules of GS, the join process is terminated; otherwise, the second process is continued. The GrC clustering algorithms are described as in Algorithm 3.

Suppose the atomic granules induced by $S$ are $g_{1}, g_{2}, g_{3}$, $g_{4}$, and $g_{5}$. The GrC clustering process can be described as the tree structure shown in Figure 4, leafs denote the atomic granules, root denotes GS including its child nodes $\mathbf{G}_{2}$ and $\mathbf{G}_{3}, \mathbf{G}_{1}$ is induced by join operation of child nodes $g_{1}$ and $g_{2}$, $\mathbf{G}_{2}$ is the join granule of $\mathbf{G}_{1}$ and $g_{3}$, and $\mathbf{G}_{3}$ is the join granule of $g_{4}$ and $g_{5}$. The whole process of obtaining GS is the bottleup process.

\section{Experiments}

Experimental settings used the same parameters in [18]; namely, the superresolution image is magnified by the input image with a factor of 3 ; for the low-resolution images, $3 \times$ 3 low-resolution patches with overlap of 1 pixel between adjacent patches and the corresponding $9 \times 9$ patches with overlap of 3 pixels for the superresolution patches are used in our experiments. The experiments include three stages: sampling, training, and reconstruction.

The sampling stage is the generation of training set for the training images in [18]. In general, the training images are SR image. The purpose of sampling stage is to form the corresponding LR image of SR image. For color images, the illuminance component is applied to the proposed algorithms since humans are more sensitive to illuminance changes. Firstly, the color image is transformed into the gray image. Secondly, LR images are extracted from SR images, SR image patches and the corresponding LR image patches are selected to form the vector, and all the vectors are used to generate the training set. 91 training images are used to form the patches to train the granule set, and 999910 patches are extracted to form the training set $\mathrm{S}$, which is redundant and has many of similar data.

The training stage is to reduce the redundancy of training set by the aforementioned $\mathrm{GrC}$ clustering. Figure 5 shows six training images with different sizes, such as flowers and faces, and the training set including redundancy patches is generated by the sampling stage. Figure 6 shows the image patches trained by $\mathrm{GrC}$ clustering with $\rho=100$.

The same reconstruction strategy as [18] is used to form the SR image in reconstruction stage. We compare SR image reconstruction via $\mathrm{GrC}$ clustering with bicubic interpolation 


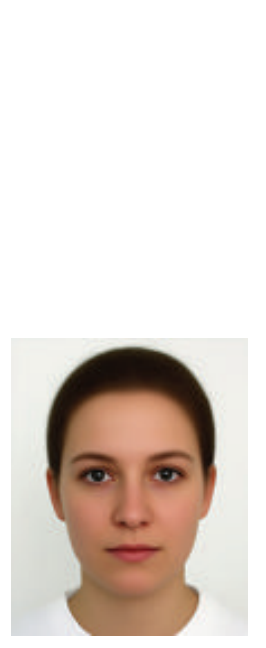

(a)

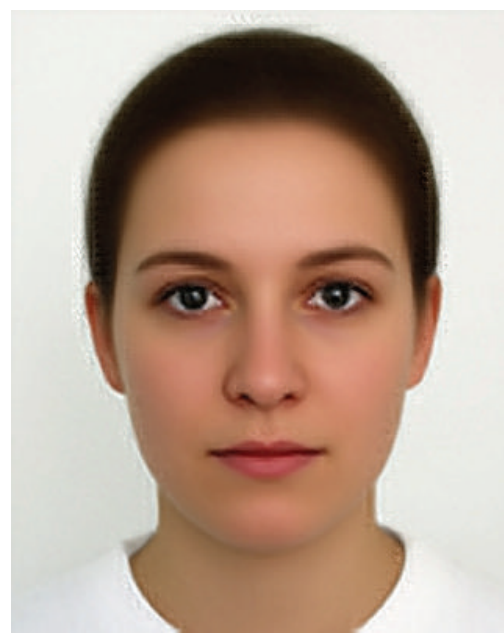

(d)

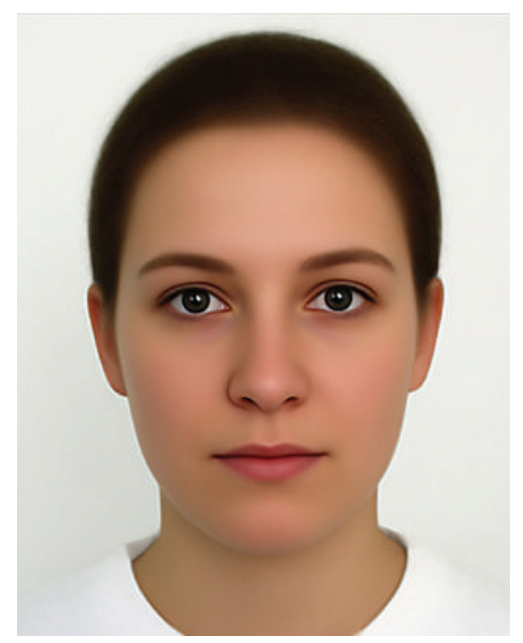

(b)

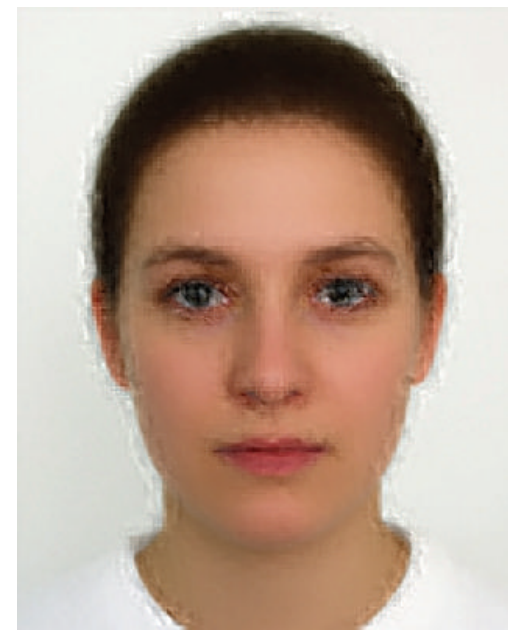

(e)

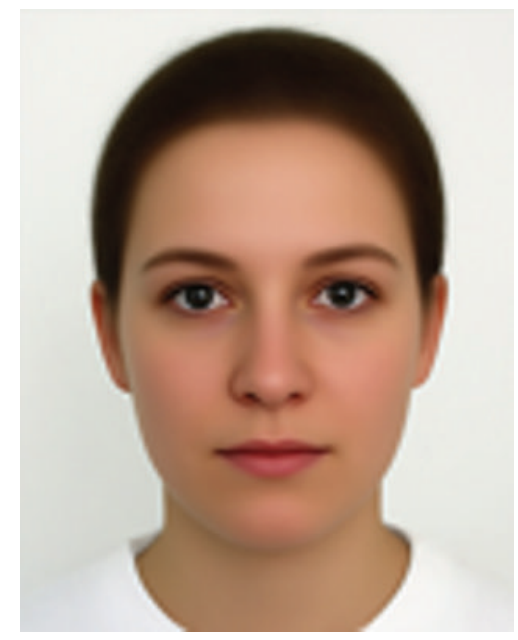

(c)

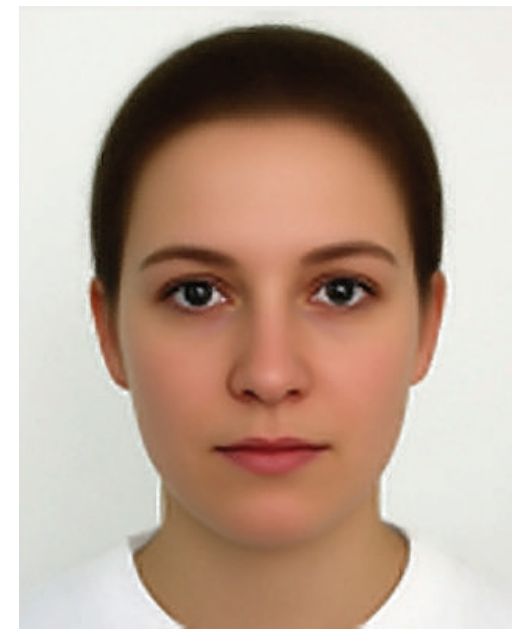

(f)

FIGURE 10: The average female face image magnified by a factor of 3. (a) Low-resolution image, (b) the original image, (c) superresolution image by bicubic interpolation, (d) superresolution image by sparse representation, (e) superresolution image by NNLasso, and (f) superresolution image by GrC.

TABLE 1: The RMSE of different methods for superresolution with magnification factor 3 with respect to the original images.

\begin{tabular}{|c|c|c|c|c|}
\hline Images & Bicubic [19] & Sparse [18] & NNLasso [20] & GrC clustering \\
\hline Flower $(330 \times 171)$ & 4.0837 & 3.9240 & 6.5283 & $3.9153(\rho=0.7)$ \\
\hline $\operatorname{Girl}(255 \times 258)$ & 6.8506 & 6.6383 & 9.9462 & $6.6318(\rho=0.7)$ \\
\hline Lenna $(512 \times 512)$ & 7.3515 & 6.9950 & 12.1007 & $6.9076(\rho=0.7)$ \\
\hline Female face $(400 \times 320)$ & 4.9347 & 4.7763 & 8.8875 & $4.4427(\rho=0.65)$ \\
\hline Male face $(400 \times 320)$ & 6.0613 & 5.7385 & 10.0067 & $5.3204(\rho=0.65)$ \\
\hline
\end{tabular}

[19], sparse representation [18], and NNLasso [20]. The performance included the SR reconstruction images and the RMSE between the SR reconstruction image and the original superresolution image.

We compared GrC clustering with sparse representation, bicubic interpolation, and NNLasso, on five test images of a flower [18], girl [18], Lenna [21], average female face [22], and average male face [22]. Firstly, training set including 999910 image patches is obtained in the sampling stage, and the redundancy of training set is reduced by $\mathrm{GrC}$ and sparse representation. Secondly, the LS images of testing images are resized by nearest method. Thirdly, the SR images are obtained by sparse representation, bicubic interpolation, NNLasso, and GrC clustering. The root mean square error (RMSE) between the superresolution images and the original images is listed in Table 1. From the table, we can see that the superresolution images by $\mathrm{GrC}$ are better than the superresolution by bicubic interpolation (bicubic), sparse 


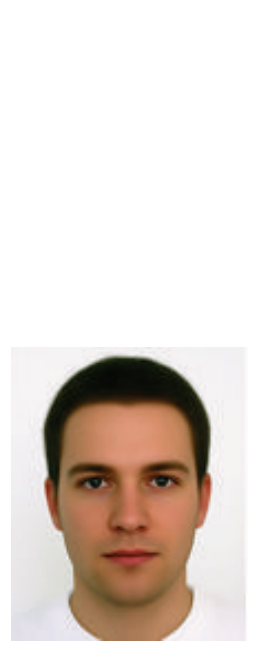

(a)

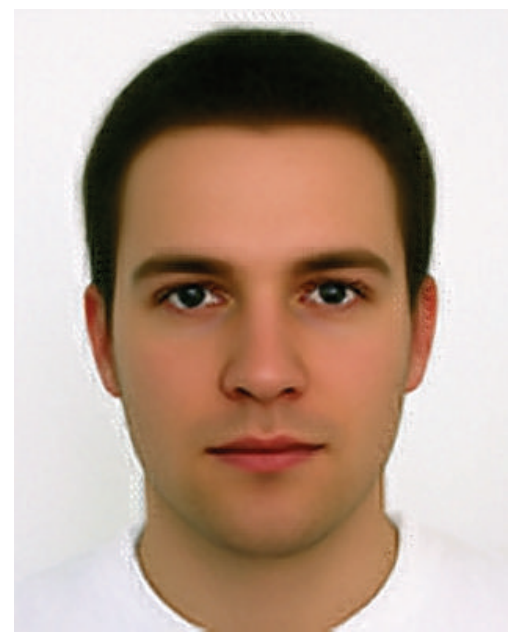

(d)

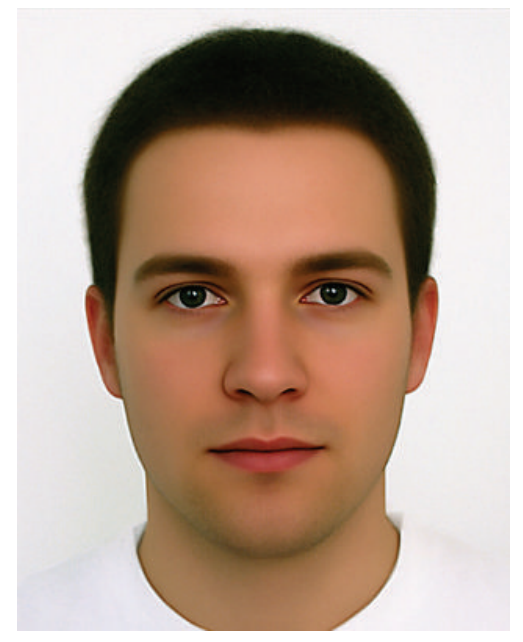

(b)

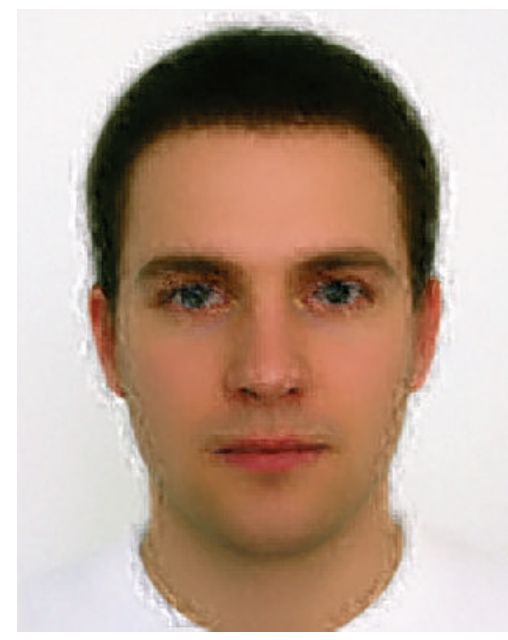

(e)

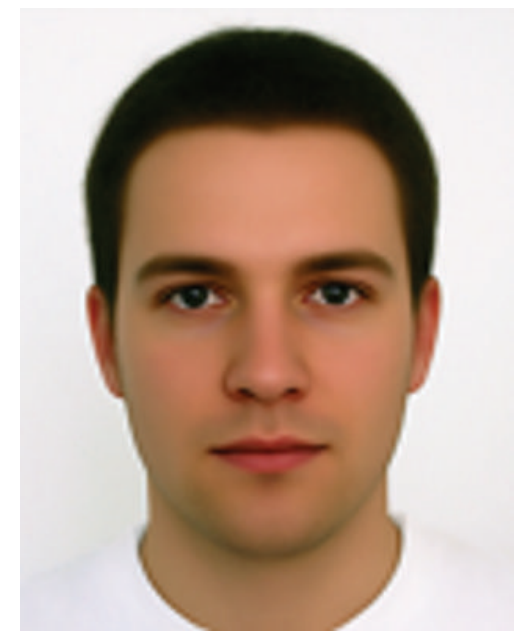

(c)

FIGURE 11: The average male face image magnified by a factor of 3. (a) Low-resolution image, (b) the original image, (c) superresolution image by bicubic interpolation, (d) superresolution image by sparse representation, (e) superresolution image by NNLasso, and (f) superresolution image by $\mathrm{GrC}$.

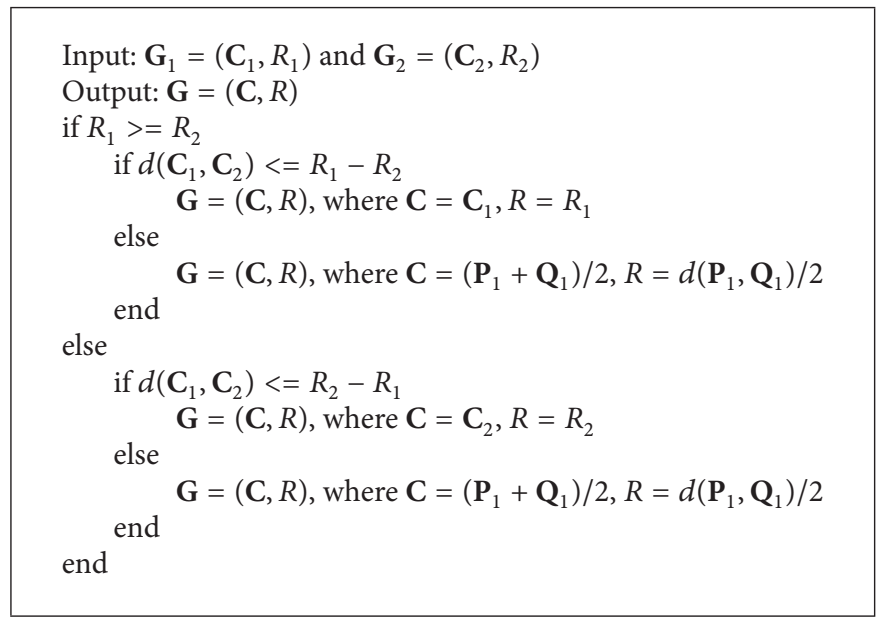

Algorithm 1: Computing $\mathbf{C}$ and $R$ of join hypersphere granule $\mathbf{G}$ between $\mathbf{G}_{1}$ and $\mathbf{G}_{2}$. 


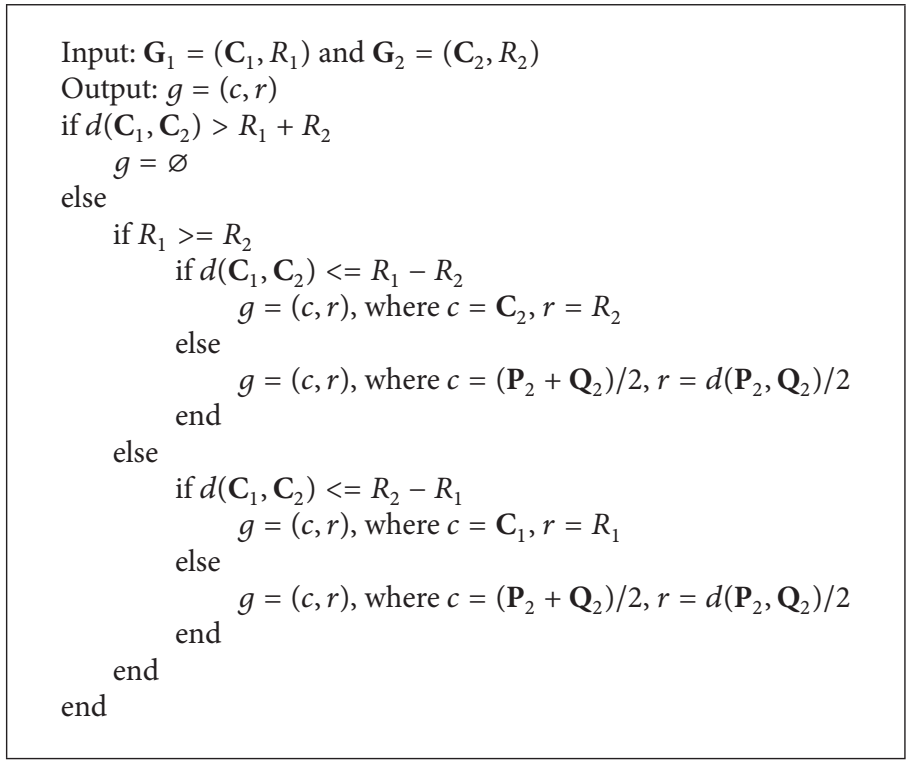

Algorithm 2: Computing $c$ and $r$ of meet hypersphere granule $g$ between $\mathbf{G}_{1}$ and $\mathbf{G}_{2}$.

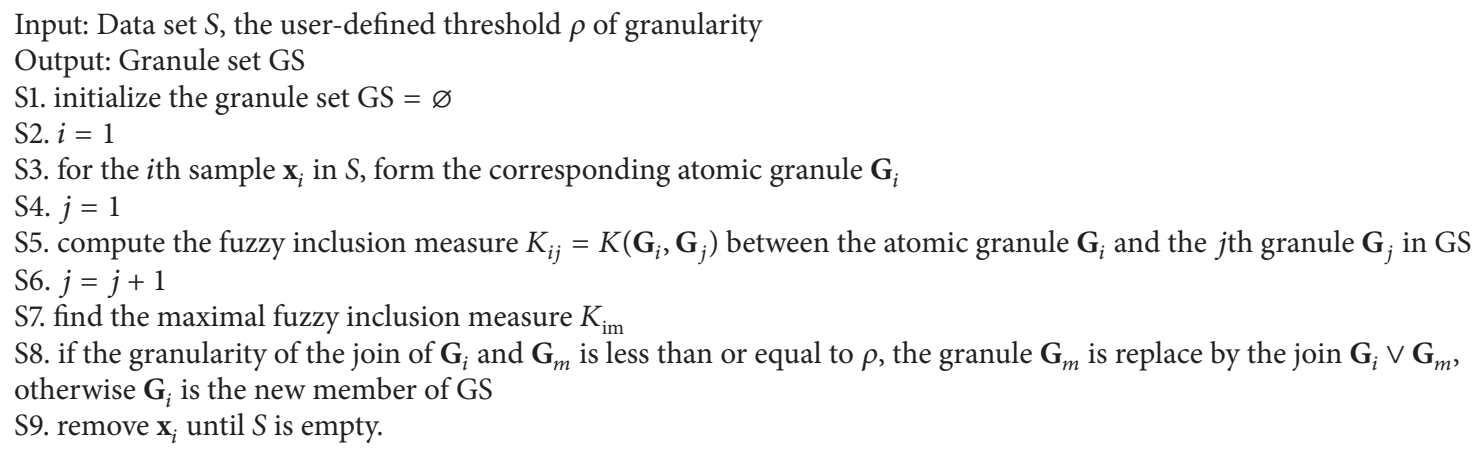

Algorithm 3: GrC clustering process.

representation (sparse), and NNLasso. The LS images, original images, and SR images are shown in Figures 7, 8, 9, 10, and 11. For human visual, the original images are the most clear, and the reconstruction images by NNLasso are blurry.

\section{Discussion}

The experimental results of the previous section demonstrate the effectiveness of image superresolution reconstruction via $\mathrm{GrC}$. However, one of the most important questions for future investigation is to determine, in terms of the within-category variation, the number of raw sample patches required to generate a dictionary satisfying $\mathrm{GrC}$. Because $\mathrm{GrC}$ is an online learning algorithm, the achieved granule set is related to the rank of training set. Image magnified by a factor of 3 is performed in the paper, and the larger magnification factors will increase the complexity of $\mathrm{GrC}$ and be discussed in the future works.

\section{Conflict of Interests}

The authors declare that there is no conflict of interests regarding the publication of this paper.

\section{Acknowledgment}

This work was supported in part by the Natural Science Foundation of China (Grant nos. 61170202 and 61202287).

\section{References}

[1] S. C. Park, M. K. Park, and M. G. Kang, "Super-resolution image reconstruction: a technical overview," IEEE Signal Processing Magazine, vol. 20, no. 3, pp. 21-36, 2003.

[2] D. Nehab, S. Rusinkiewicz, and J. Davis, "Improved subpixel stereo correspondences through symmetric refinement," in Proceedings of the 10th IEEE International Conference on Computer Vision (ICCV '05), vol. 1, pp. 557-563, Beijing, China, October 2005. 
[3] A. N. Benaichouche, H. Oulhadj, and P. Siarry, "Improved spatial fuzzy c-means clustering for image segmentation using PSO initialization, Mahalanobis distance and post-segmentation correction," Digital Signal Processing, vol. 23, no. 5, pp. 13901400, 2013.

[4] R. Fleysher, L. Fleysher, S. Liu, and O. Gonen, "On the voxel size and magnetic field strength dependence of spectral resolution in magnetic resonance spectroscopy," Magnetic Resonance Imaging, vol. 27, no. 2, pp. 222-232, 2009.

[5] C. V. Rao, K. M. M. Rao, P. S. Reddy, and G. Pujar, "A novel method for enhancement of radiometric resolution using image fusion," International Journal of Applied Earth Observation and Geoinformation, vol. 10, no. 2, pp. 165-174, 2008.

[6] S. Villena, M. Vega, R. Molina, and A. K. Katsaggelos, "A nonstationary image prior combination in super-resolution," Digital Signal Processing, vol. 32, no. 1, pp. 1-10, 2014.

[7] R. M. Bahy, G. I. Salama, and T. A. Mahmoud, "Adaptive regularization-based super resolution reconstruction technique for multi-focus low-resolution images," Signal Processing, vol. 103, no. 1, pp. 155-167, 2014.

[8] L. A. Zadeh, Fuzzy Sets and Information Granulation, North Holland Publishing, 1979.

[9] L. A. Zadeh, "Toward a theory of fuzzy information granulation and its centrality in human reasoning and fuzzy logic," Fuzzy Sets and Systems, vol. 90, no. 2, pp. 111-127, 1997.

[10] V. G. Kaburlasos and S. E. Papadakis, "A granular extension of the fuzzy-ARTMAP (FAM) neural classifier based on fuzzy lattice reasoning (FLR)," Neurocomputing, vol. 72, no. 10-12, pp. 2067-2078, 2009.

[11] V. G. Kaburlasos, L. Moussiades, and A. Vakali, "Fuzzy lattice reasoning (FLR) type neural computation for weighted graph partitioning," Neurocomputing, vol. 72, no. 10-12, pp. 2121-2133, 2009.

[12] V. G. Kaburlasos, Towards a Unified Modeling and KnowledgeRepresentation Based on Lattice Theory-Computational Intelligence and Soft Computing Applications, vol. 27 of Studies in Computational Intelligence, Springer, Heidelberg, Germany, 2006.

[13] V. G. Kaburlasos, S. E. Papadakis, and G. A. Papakostas, "Lattice computing extension of the fam neural classifier for human facial expression recognition," IEEE Transactions on Neural Networks and Learning Systems, vol. 24, no. 10, pp. 1526-1538, 2013.

[14] V. G. Kaburlasos and L. Moussiades, "Induction of formal concepts by lattice computing techniques for tunable classification," Journal of Engineering Science and Technology Review, vol. 7, no. 1, pp. 1-8, 2014.

[15] V. G. Kaburlasos and A. Kehagias, "Fuzzy inference system (FIS) extensions based on lattice theory," IEEE Transactions on Fuzzy Systems, vol. 22, no. 3, pp. 531-546, 2014.

[16] P. Sussner, M. Nachtegael, T. Mélange, G. Deschrijver, E. Esmi, and E. Kerre, "Interval-valued and intuitionistic fuzzy mathematical morphologies as special cases of $L$-fuzzy mathematical morphology," Journal of Mathematical Imaging and Vision, vol. 43, no. 1, pp. 50-71, 2012.

[17] G. Birkhoff, Lattice Theory, American Mathematical Society, Providence, RI, USA, 1967.

[18] J. Yang, J. Wright, T. S. Huang, and Y. Ma, "Image superresolution via sparse representation," IEEE Transactions on Image Processing, vol. 19, no. 11, pp. 2861-2873, 2010.
[19] R. G. Keys, "Cubic convolution interpolation for digital image processing," IEEE Transactions on Acoustics, Speech, and Signal Processing, vol. 29, no. 6, pp. 1153-1160, 1981.

[20] R. Tibshirani, "Regression shrinkage and selection via the lasso," Journal of the Royal Statistical Society Series B, vol. 58, no. 1, pp. 267-288, 1996.

[21] http://www.cs.cmu.edu/ chuck/lennapg.

[22] http://www.facedetection.com/facedetection/datasets.htm. 

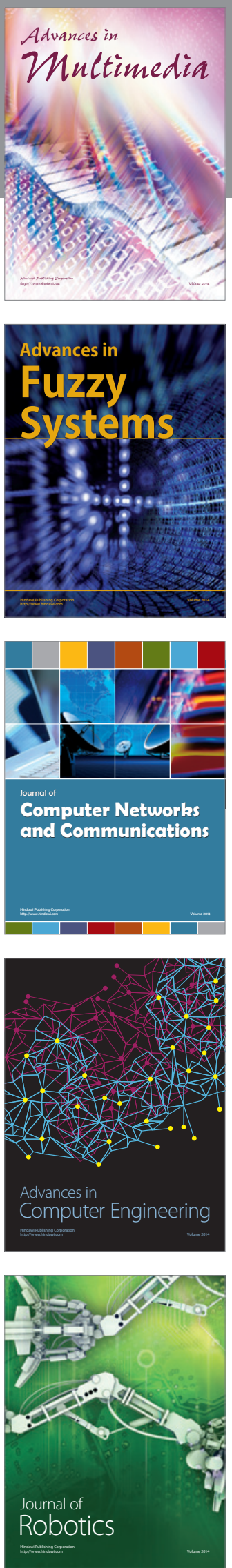

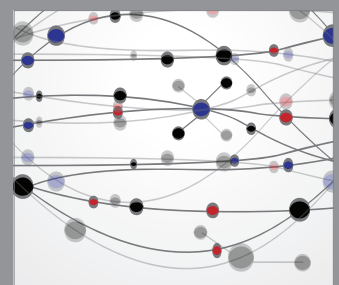

The Scientific World Journal
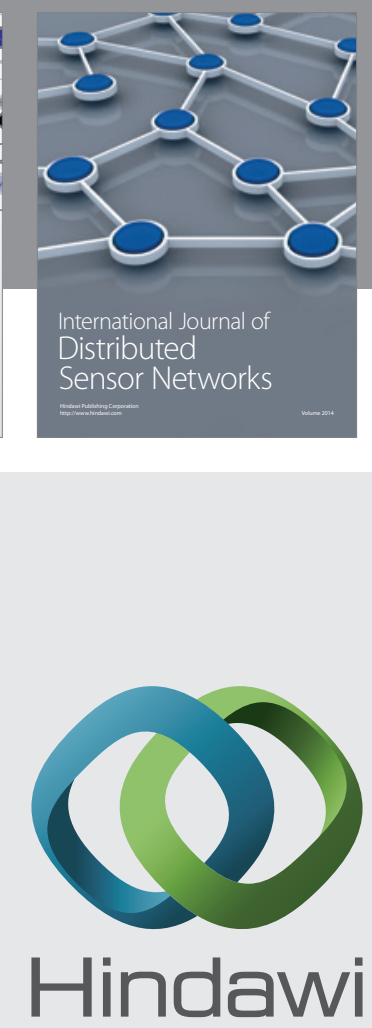

Submit your manuscripts at

http://www.hindawi.com
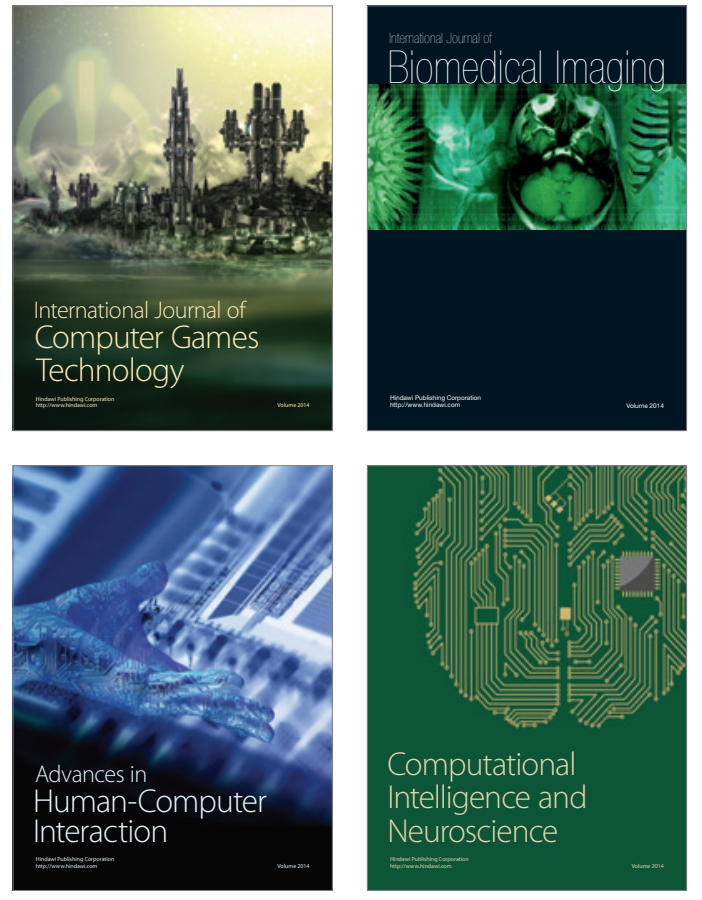
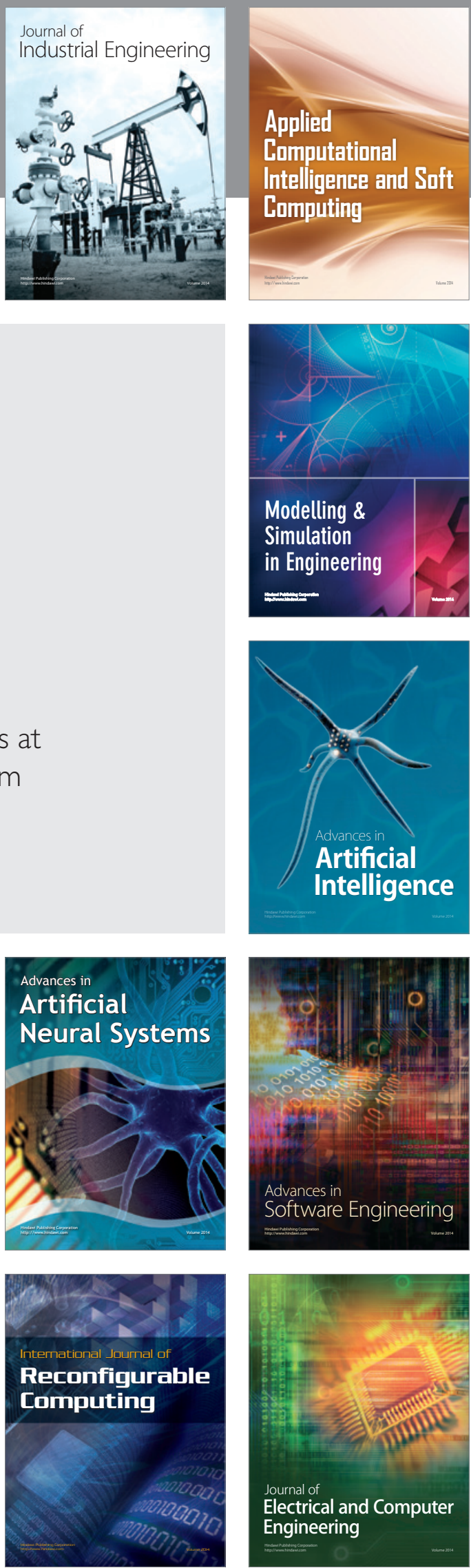